SUPPORTING INFORMATION

\title{
Gold Nanostar Spatial Distribution Impacts the Surface-Enhanced Raman Scattering Detection of Uranyl on Amidoximated Polymers
}

Hoa T. Phan, Claire Vinson, and Amanda J. Haes*

Department of Chemistry, University of Iowa, Iowa City, IA 52242

*Email: amanda-haes@uiowa.edu; Tel: 319-384-3695

$\underline{\text { Table of Contents }}$

Fabrication, Functionalization, and Characterization of Electrospun Polymers....................... S2

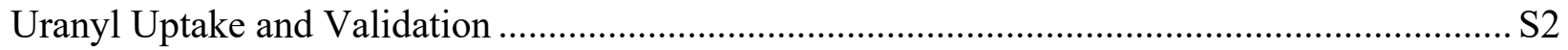

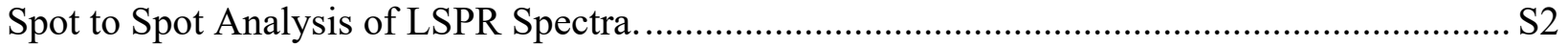

LSPR Map of Traditional, Upright Nanostar Deposition. ................................................... S3

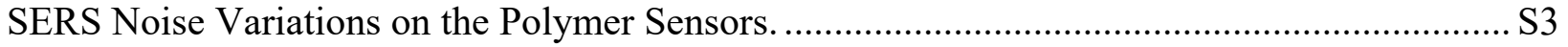

Vibrational Mode Assignments for Uranyl Species ............................................................ S3 
Fabrication, Functionalization, and Characterization of Electrospun Polymers. Polymers were fabricated according to previously published procedures. ${ }^{1-2}$ Briefly, a polyvinylidene fluoride (PVDF) layer was electrospun onto a grounded drum collector, followed by a polyacrylonitrile (PAN) layer. The polymer film was then removed from the drum and amidoximated in a solution containing hydroxylammonium chloride $(15 \mathrm{~g} / \mathrm{L})$ and sodium hydroxide $(15 \mathrm{~g} / \mathrm{L})$ for 24 hours at $30^{\circ} \mathrm{C}$. The mass-to-volume ratio of the polymer to amidoxime solution was maintained at $1 \mathrm{mg} / \mathrm{mL}$. The polymers were then rinsed with DI water, flattened onto a polystyrene petri dish with the PAN side up, dried for 12 hours under standard room conditions, and stored until use.

Uranyl Uptake and Validation. Uranyl uptake was quantified using a previously published LSC protocol ${ }^{1}$ based on the amount of ${ }^{238} \mathrm{U}$ in solution before and after polymer incubation as well as after its dissolution. ${ }^{232} \mathrm{U}$ radiotracer (3.5 Bq, NIST traceable standard, Eckert \& Ziegler) was also used to increase the signal-to-noise ratios for these measurements.

Spot to Spot Analysis of LSPR Spectra. Representative LSPR spectra of gold nanostars deposited on sensor substrates using an inverted drop coating method are shown in Figure S1. Although these spectra are collected inside the nanostar coffee ring, fluctuation in spectral background is observed thereby limiting nanostar quantification. Corresponding second derivative processing eliminates changes in the spectral background thereby facilitating quantification of local nanostar surface density as illustrated in Figure 4A.

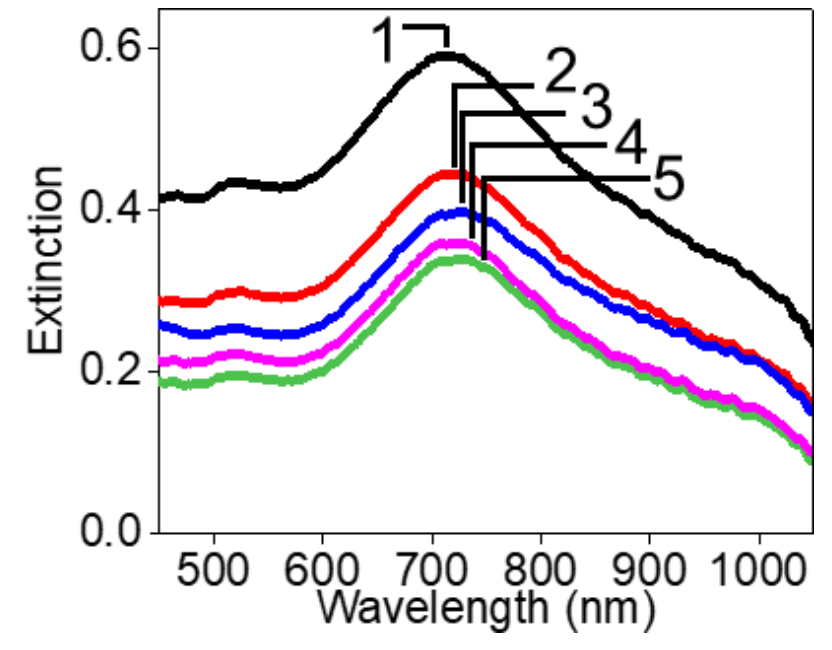

Figure S1. LSPR spectra collected at various spectral locations on a representative polymeric substrates. 
LSPR Map of Traditional, Upright Nanostar Deposition. The optical density of gold nanostar multilayers on the polymer substrate is calculated using eq. 1. A secondderivative extinction magnitude at the $\lambda_{\max }$ is divided by the second-derivative extinction coefficient of $1.80 \times 10^{5} \mathrm{M}^{-1}$ and then multiplied by 3.6. This allows for the generation of a correction factor for scattering losses. The LSPR map for gold nanostar multilayers deposited on the polymer substrate using the upright geometry is shown in Figure S2. The optical density varies from 725 to $1013 \mathrm{pM} \cdot \mathrm{cm}($ average $=853 \pm 70(8 \%$ RSD), median $=850$ ) over a $1 \times 1 \mathrm{~mm}^{2}$ area.

SERS Noise Variations on the Polymer

Sensors. Noise was calculated from 770 to $700 \mathrm{~cm}-1$ in SERS spectra collected on the same substrates shown in Figures 5B and 5C for the upright and inverted geometries, respectively. A spatial comparison of noise from these samples is shown in Figure S3.

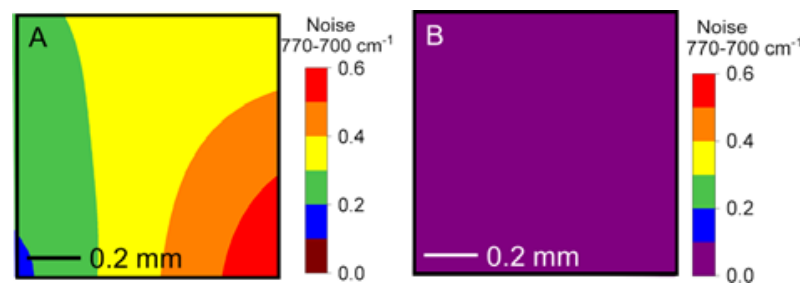

Figure S3. Spatial distribution of noise on polymeric substrates using (A) upright droplet and (B) inverted deposition geometries. These regions are the same as those shown in Figure $5 \mathrm{~B}$ and $5 \mathrm{C}$.

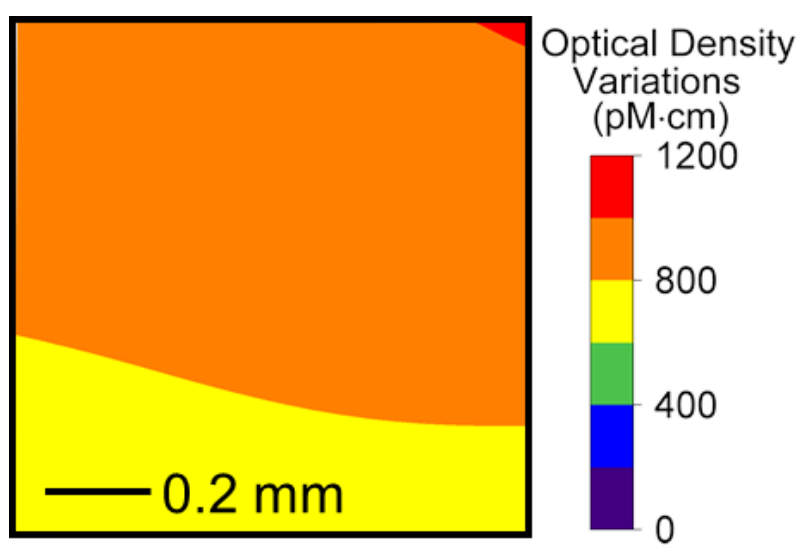

Figure S2. Spatial distribution of local nanoparticle optical density on a $1 \times 1 \mathrm{~mm}^{2}$ area in the center of the polymer substrate generated using an upright droplet deposition geometry. Experimental parameters: 20x objective and transmission geometry.
Vibrational Mode Assignments for Uranyl Species. Three uranyl-specific vibrational modes are detected in SERS. These are centered at $846 \pm 1(\Gamma=12 \pm 1), 833 \pm 1$ $(13 \pm 1)$, and $821 \pm 1\left(17 \pm 1 \mathrm{~cm}^{-1}\right) \mathrm{cm}^{-1}$. To assign these features to species, we first consider only the water-soluble uranyl species that should be present initially. These were determined using Hydra and Medusa, ${ }^{3}$ a chemical database that contains species and their formation constants to generate chemical equilibrium diagrams.

In this study, $\mathrm{pH}=6.8$ and ionic strength $=$

$3 \mathrm{mM}$ are used to mimic relevant experimental conditions. The relative abundance of soluble uranyl species that are predicted to initially present are shown in Figure S4. Because coordination to amidoxime is disrupted by carboxylate, ${ }^{4}$ we next consider impacts of monodentate and bidentate carboxylate ligand coordination to these soluble species. Previously, these were shown to induce $\sim 13$ and $\sim 24 \mathrm{~cm}^{-1}$ red-shifts in the vibrational frequencies, respectively. ${ }^{4}$ Additionally, with the dominant concentration of carboxylate vs. hydroxide on the polymer surface, carboxylate can also replace hydroxide to coordinate with uranyl. As a result, the vibrational features centered at 846,



Figure S4. Fraction of uranyl complexes in $10 \mathrm{mM}$ HEPES (pH 6.8). 
833, and 821 are assigned as $\mathrm{UO}_{2}(\text { bidentate- } \mathrm{COO})^{+}, \mathrm{UO}_{2}(\mathrm{OH})($ monodentate- $\mathrm{COO})$, and $\left(\mathrm{UO}_{2}\right)_{3}(\mathrm{OH})_{5}($ monodentate- $\mathrm{COO}) / \mathrm{UO}_{2}(\mathrm{OH})($ bi-COO$)$, respectively, and are summarized in Table S1.

Table S1. SERS Band Assignments of Observed Uranyl Species.

\begin{tabular}{|c|c|c|c|c|}
\hline \multicolumn{2}{|c|}{ Raman } & \multicolumn{2}{c|}{ SERS } & \multirow{2}{*}{$\begin{array}{c}\bar{v}_{\text {Raman }}-\bar{v}_{S E R S} \\
\left(\Delta \mathrm{cm}^{-1}\right)\end{array}$} \\
\hline Species & $\bar{v}\left(\Delta \mathrm{cm}^{-1}\right)$ & Complex & $\bar{v}\left(\Delta \mathrm{cm}^{-1}\right)$ & $24 \pm 1$ \\
\hline $\mathrm{UO}_{2} \mathrm{OH}^{+}$ & $848 \pm 2^{3,5-6}$ & $\mathrm{UO}_{2}(\mathrm{bi}-\mathrm{COO})^{+}$ & $846 \pm 1$ & $15 \pm 2$ \\
\cline { 3 - 4 } & & $\mathrm{UO}_{2} \mathrm{OH}($ mono-COO $)$ & $833 \pm 1$ & $14 \pm 1$ \\
\hline$\left(\mathrm{UO}_{2}\right)_{3}(\mathrm{OH})_{5}^{+}$ & $835 \pm 1^{3,5-6}$ & $\left(\mathrm{UO}_{2}\right)_{3}(\mathrm{OH}) 5(\mathrm{mono}-\mathrm{COO})$ & $821 \pm 1$ & $5 \pm 2$ \\
\hline $\mathrm{UO}_{2}(\mathrm{OH})_{2}$ & $826 \pm 2^{3,5-6}$ & $\mathrm{UO}_{2}(\mathrm{OH})(\mathrm{bi}-\mathrm{COO})$ & $821 \pm 1$ & \\
\hline
\end{tabular}

\section{REFERENCES}

1. Lu, G.; Johns, A. J.; Neupane, B.; Phan, H. T.; Cwiertny, D. M.; Forbes, T. Z.; Haes, A. J., Matrix-Independent Surface-Enhanced Raman Scattering Detection of Uranyl Using Electrospun Amidoximated Polyacrylonitrile Mats and Gold Nanostars. Anal. Chem. 2018, 90 (11), 6766-6772. 2. Johns, A.; Qian, J.; Carolan, M. E.; Shaikh, N.; Peroutka, A.; Seeger, A.; Cerrato, J. M.; Forbes, T. Z.; Cwiertny, D. M., Functionalized Electrospun Polymer Nanofibers for Treatment of Water Contaminated with Uranium. Environ. Sci.: Water Res. Technol. 2020, 6 (3), 622-634.

3. Lu, G.; Forbes, T. Z.; Haes, A. J., Evaluating Best Practices in Raman Spectral Analysis for Uranium Speciation and Relative Abundance in Aqueous Solutions. Anal. Chem. 2016, 88 (1), 773-780.

4. Quilès, F.; Burneau, A., Infrared and Raman Spectroscopic Study of Uranyl Complexes: Hydroxide and Acetate Derivatives in Aqueous Solution. Vib. Spectrosc. 1998, 18 (1), 61-75.

5. $\quad$ Nguyen Trung, C.; Begun, G. M.; Palmer, D. A., Aqueous Uranium Complexes. 2. Raman Spectroscopic Study of The Complex Formation of The Dioxouranium(VI) Ion with a Variety of Inorganic and Organic Ligands. Inorg. Chem. 1992, 31 (25), 5280-5287.

6. $\quad$ Nguyen-Trung, C.; Palmer, D. A.; Begun, G. M.; Peiffert, C.; Mesmer, R. E., Aqueous Uranyl Complexes 1. Raman Spectroscopic Study of the Hydrolysis of Uranyl(VI) in Solutions of Trifluoromethanesulfonic Acid and/or Tetramethylammonium Hydroxide at $25^{\circ} \mathrm{C}$ and $0.1 \mathrm{MPa} . J$. Solution Chem. 2000, 29 (2), 101-129. 